\title{
Minute Times Mole per Liter per Kilogram per Meter Squared
}

National Cancer Institute

\section{Source}

National Cancer Institute. Minute Times Mole per Liter per Kilogram per Meter Squared. NCI Thesaurus. Code C111266.

Minutes times moles per liter, divided by kilograms per meter squared. 